\section{SANDIA REPORT}

SAND98-2269

Unlimited Release

Printed January 1999
1-MS-0619-Roview\&-Approvat-Besk

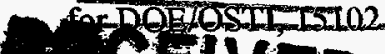
FEB 051909 OST,

\title{
Z-pinch Driven Isentropic Compression for Inertial Fusion
}

S. A. Slutz, R. B. Spielman, W. A. Stygar, J. R. Asay, C. A. Hall, and K. G. Holland

Prepared by

Sandia National Laboratories

Aibuquerque, New Mexico 87185 and Livermore, California 94550

Sandia is a multiprogram laboratory operated by Sandia Corporation, a Lockheed Martin Company. for the United States Department of Energy under Contract DE-AC04-94AL85000.

Approved for public release, further dissemination unlimited.

\section{Sandia National Laboratories}


Issued by Sandia National Laboratories, operated for the United States Department of Energy by Sandia Corporation.

NOTICE: This report was prepared as an account of work sponsored by an agency of the United States Government. Neither the United States Government, nor any agency thereof, nor any of their employees, nor any of their contractors, subcontractors, or their employees, make any warranty, express or implied, or assume any legal liability or responsibility for the accuracy, completeness, or usefulness of any information, apparatus, product, or process disclosed, or represent that its use would not infringe privately owned rights. Reference herein to any specific commercial product, process, or service by trade name, trademark, manufacturer, or otherwise, does not necessarily constitute or imply its endorsement, recommendation, or favoring by the United States Government, any agency thereof, or any of their contractors or subcontractors. The views and opinions expressed herein do not necessarily state or reflect those of the United States Government, any agency thereof, or any of their contractors.

Printed in the United States of America. This report has been reproduced directly from the best available copy.

Available to DOE and DOE contractors from

Office of Scientific and Technical Information

P.O. Box 62

Oak Ridge, TN 37831

Prices available from (703) 605-6000

Web site: http://www.ntis.gov/ordering.htm

Available to the public from

National Technical Information Service

U.S. Department of Commerce

5285 Port Royal Rd

Springfield, VA 22161

NTIS price codes

Printed copy: A03

Microfiche copy: A0I

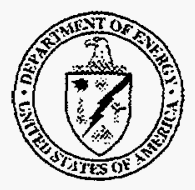




\section{DISCLAIMER}

Portions of this document may be illegible in electronic image products. Images are produced from the best available original document. 
SAND98-2269

Unlimited Release

Printed January 1999

\title{
Z-pinch Driven Isentropic Compression for Inertial Fusion
}

\author{
S. A. Slutz \\ Target and Z-Pinch Theory Department \\ R. B. Spielman, W. A. Stygar \\ Load Coupling and Z-Pinch Source Development Department \\ J. R. Asay, C. A. Hall, K. G. Holland \\ Shock Physics Application Department \\ Sandia National Laboratories \\ P. O. Box 5800 \\ Albuquerque, NM 87185-1186
}

\begin{abstract}
The achievement of high gain with inertial fusion requires the compression of hydrogen isotopes to high density and temperatures. High densities can be achieved most efficiently by isentropic compression. This requires relatively slow pressure pulses on the order of 10-20 nanoseconds; however, the pressure profile must have the appropriate time history [J. Nuckolls et. al. Nature, 239, 139, 1972]. We present 1-D numerical simulations that indicate such a pressure profile can be generated by using pulsed power driven $z$ pinches. Although high compression is calculated, the fuel temperature is too low for ignition. Ignition could be achieved by heating a small portion of this compressed fuel with a short (-10 ps) high power laser pulse as previously described [M. Tabak et. al. Phys. Plasmas 1, 1626, 1994]. Our 1-D calculations indicate that the existing Z-accelerator could provide the driving current ( 20 MA) necessary to compress fuel to roughly 1500 times solid density. At this density the required laser energy is approximately $10 \mathrm{~kJ}$. Multidimensional effects such as the Rayleigh-Taylor were not addressed in this brief numerical study. These effects will undoubtedly lower fuel compression for a given drive current. Therefore it is necessary to perform z-pinch driven compression experiments. Finally, we present preliminary experimental data from the Z-accelerator indicating that current can be efficiently delivered to appropriately small loads ( $\sim 5 \mathrm{~mm}$ radius) and that VISAR can be used measure high pressure during isentropic compression.
\end{abstract}




\section{acknowledgments}

We gratefully acknowledge the experimental support of Tim Wagoner and all of the Zcrew. We also thank Ken Struve for theoretical support and data analysis. 


\section{Contents}

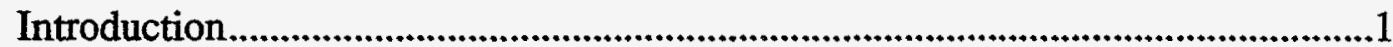

Magnetic inhibition of alpha particle transport.........................................................3

Quasi-spherical isentropic z-pinch compression .....................................................8

Experimental plans and preliminary results...........................................................15

Conclusions and future work ....................................................................19

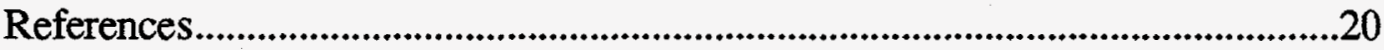

\section{Figures}

The minimum $\rho_{0} r_{0}$ is plotted as a function of $\Delta=\rho_{d} / \rho_{0}$ .6

A schematic of a quasi-spherical compression .........................................8

Flux compression geometry

Calculated current waveforms ..........................................................12

A schematic cross section of the load region.

Current wave forms for shot 315 


\section{Introduction}

The achievement of high gain with inertial confinement fusion (ICF) requires the compression of hydrogen isotopes to high density and temperatures. High densities are needed so that a significant fraction of the nuclear fuel has fused before the target disassembles. High densities can be achieved most efficiently when the compression does not increase entropy or the compression is isentropic. During isentropic compression the pressure will be approximately described by the relation

$$
\mathrm{P}=\mathrm{P}_{0}(\mathrm{~S})\left(\frac{\rho}{\rho_{0}}\right)^{\gamma}
$$

where $\gamma=5 / 3$ for an ideal or a Fermi degenerate gas, $\rho$ is the density, $P_{0}$ and $\rho_{0}$ are the initial pressure and density. The initial pressure depends of the entropy of the material being compressed. The function $P_{0}(S)$ is monotonically increasing with the entropy, $S$. Thus the most efficient implosion maintains the entropy at the minimum value. Since heat input or shock waves increase the entropy these must be avoided. A strong shock wave will be formed if the pressure rises too quickly. The length of the pulse depends on the size of the system. Typically ICF capsules have radii one to several $\mathrm{mm}$, and the pressure pulse must be from one to several tens of nanoseconds. Furthermore, the pressure profile must have the appropriate time history ${ }^{1}$. At the beginning of the pulse the pressure is only slightly greater than the strength of the material being compressed. Typically this is approximately 1 Megabar $\left(\sim 10^{5} \mathrm{~Pa}\right)$. A weak shock wave is launched into the material that compresses the material without a significant increase in the entropy. The driving pressure can then be increased launching another weak shock, which will propagate at a faster velocity than the first. All of these weak shocks can arrive at the center of the target at the same time resulting very high compression when an appropriate pressure profile is used. We present calculations indicating that such a pressure profile can be accomplished using pulsed power driven $\mathrm{z}$ pinches. However, the fuel remains relatively cold during the compression. 
A small portion of the fuel could be raised to the high temperatures needed for ignition $(>4 \mathrm{keV})$ with a short high power laser pulse $(\sim 10 \mathrm{ps})$ as described by Max Tabak et. al. ${ }^{2}$. Only a portion of the fuel needs to be heated, since this "hot spot" will undergo fusion (burn) and ignite the cold fuel surrounding it. Under the proper conditions a fusion detonation wave travels through all of the cold fuel. A target ignited in this manner yields high gain, since most of the fuel does not have to be raised to ignition temperatures directly. The energy required to heat the hot spot depends linearly on the hot spot mass, which is determined by the requirement that a significant fraction of the alpha particles produce by the fusion reaction are deposited within the hot spot ${ }^{2}$. Thus the radius of the hot spot, $\mathrm{r}_{\mathrm{hs}}<$ $R_{\alpha} / \rho$, where the range of an alpha particle, $R_{\alpha} \sim 0.4 \mathrm{gm} / \mathrm{cm}^{2}$. The hot spot mass is $M_{h s}=$ $(4 / 3) \pi R_{\alpha}^{3} / \rho^{2}$. The temperature of the hot spot must be raised above the ideal ignition temperature. Assuming $3 \mathrm{kT} / 2$ of energy/particle at $10 \mathrm{keV}$ the minimum hot spot energy is $\mathrm{E}_{\mathrm{hs}}=320 / \mathrm{\rho}^{2} \mathrm{MJ}$. This yields approximately $3 \mathrm{~kJ}$ at a density 1200 times the solid density of frozen deuterium-tritium (DT). Numerical simulations indicate ${ }^{3}$ that hot electrons can be generated at about $40 \%$ efficiency by a high intensity laser, $\mathrm{I}^{2}=10^{20} \mathrm{~W} \mathrm{cm^{-2 }} \mu \mathrm{m}^{2}$. Thus at this density approximately $7.5 \mathrm{~kJ}$ of laser energy would be required. This laser energy must be delivered in a time less than the hot spot disassembly time, $\tau_{D} \sim R_{\alpha} / c_{s} \rho$, which is about 10 ps at $\rho=300 \mathrm{gm} / \mathrm{cm}^{2}$.

Thus laser energy required for ignition $(\sim 10 \mathrm{~kJ})$ is much less than the energy required for compression (> $1 \mathrm{MJ}$ ). Consequently, the cost of driving inertial fusion could be reduced significantly by combining these two technologies.

The natural geometry of z-pinch implosions is cylindrical. The convergence ratio $\eta=$ $\mathrm{I}_{0} / \mathrm{r}$ is limited by the Rayleigh-Taylor instability. Values of $10-20$ are generally believed to be possible for a liner implosion. Such values have been attained in wire array implosions. Even assuming $\eta=20, E_{\mathrm{hs}} \sim 40 \mathrm{~kJ}$, which would require approximately $100 \mathrm{~kJ}$ of laser energy. This is a rather large laser. Quasi-spherical implosions have the potential to 
increase the final density for a given compression ratio and could reduce the required laser energy. However, such an implosion is more complicated than a purely cylindrical implosion. In section II we consider the application of a strong magnetic field to inhibit the transport of the alpha particles, which could potentially lower the hot spot energy requirement. Although the gain within the hot spot is improved by the presence of the magnetic field, the propagation of the burn transverse to the magnetic field into the surrounding cold fuel is inhibited. The net result is that introducing the magnetic field does not significantly lower the hot spot energy needed to obtain propagating burn. In section III we consider quasi-spherical z-pinch implosions. We present ideal 1-D calculations yielding compressed fuel densities of $300 \mathrm{gm} / \mathrm{cm}^{2}$, with an accelerator drive current of only $20 \mathrm{MA}$. Of course 2-D effects will degrade this performance, but the approach looks promising. In section IV, we discuss a sequence of experiments to determine how effectively $\mathrm{z}$ pinches can compress material and preliminary experimental results are presented. Finally, our results are summarized in section $\mathrm{V}$.

\section{Magnetic inhibition of alpha particle transport}

A strong magnetic field can be used to decrease the effective alpha particle range. The cyclotron radius of a $3.5 \mathrm{MeV}$ alpha particle is $r_{c \alpha}=53 / \mathrm{B} \mathrm{cm}$ with B in Tesla. A very strong axial magnetic field could be produced in a cylindrical system by imposing a strong slow field (B 1 MG) and then compressing the cylinder with an axial current. Lasnex ${ }^{4}$ has been used to calculate the implosion of such a cylindrical liner system. The simulations indicated that DT densities of up to $50 \mathrm{gm} / \mathrm{cm}^{3}$ could be achieved with a drive current that rises linearly up to a maximum value of $20 \mathrm{MA}$ in $200 \mathrm{~ns}$. The liner consisted of solid DT ( $\rho=0.25 \mathrm{gm} / \mathrm{cm} 3$ ) out to a radius of $3 \mathrm{~mm}$, surrounded by a $0.3 \mathrm{~mm}$ layer of aluminum. The initial magnetic field of 1 MG was increased to greater than 200 MG. After compression, a laser aimed along the axis could heat a small rod shaped region. Since the $\mathrm{B}$-field is in the z-direction, it does not significantly effect the alpha propagation in the z- 
direction. Thus the cylinder must be long enough so that $p z>R_{\alpha}$. Once the hot spot ignites, the burn would propagate in the radial direction. The mass of this hot spot is then $M_{h s}=\rho z \pi r_{c \alpha}^{2}$. The energy required to heat the hot spot to $10 \mathrm{keV}$ is then

$$
\mathrm{E}_{\mathrm{hs}}=\frac{4.2 \times 10^{12}}{\mathrm{~B}_{0}^{2} \eta^{4}} \text { joules }
$$

where $B_{0}$ is the initial magnetic field. Assuming $B_{0}=200 \mathrm{~T}$, and $\eta=15$, we obtain $E_{h s}=$ $4.1 \mathrm{~kJ}$, which would require about $10 \mathrm{~kJ}$ of laser energy. If this was the whole story, introducing a magnetic field would dramatically reduce the laser energy required for ignition at low fuel densities. However, the confinement time of the hot spot must also be considered. Efficient burn of the hot spot fuel requires significant self heating. To estimate the degree of self heating, we use the approximate relation

$$
f_{\alpha} \approx\left(\frac{r_{0}}{r_{0}+r_{\alpha}}\right)^{2}
$$

where $f_{\alpha}$ is the fraction of the alpha particles that are deposited within the hot spot, $r_{0}$ is the initial radius of the hot spot, and $r_{\alpha}=\min \left(r_{c \alpha}, \frac{R_{\alpha}}{\rho}\right)$. The fusion power into alpha particle heating of the hot spot is

$$
\mathrm{W}_{\alpha}=\mathrm{f}_{\alpha} \mathrm{W}_{\mathrm{b}} \rho^{2},
$$

where $\mathrm{W}_{\mathrm{b}}=3.6 \times 10^{18}$ watts- $\mathrm{cm}^{3} / \mathrm{gm}^{2}$. The presence of the strong magnetic field suppresses conduction losses so the dominant loss is due to hydrodynamic work

$$
\mathrm{W}_{\mathrm{h}}=\frac{\mathrm{Pv}}{\mathrm{r}},
$$

where $P$ is the pressure, $v$ is the velocity, and $r$ is the radius of the hot spot. The hot spot is confined as long as the alpha heating exceeds the hydrodynamic losses. Thus combining eqs (3)-(5), we obtain the condition 


$$
\mathrm{ux} \leq \mathrm{f}_{\alpha} \mathrm{W}_{\mathrm{b}} \rho_{0} \mathrm{r}_{0}\left(\frac{\rho_{0}}{2 \mathrm{P}_{0}}\right)^{3 / 2} \approx 6.3 \mathrm{f}_{\alpha} \rho_{0} \mathrm{r}_{0},
$$

where $u=v\left(5 m_{p} / 6 k T\right)^{1 / 2}, m_{p}$ is the mass of a proton, and $x=r / r_{0}$. Since the hot spot pressure is much larger than the pressure of the surrounding cold fuel, the hot spot will expand into the cold fuel. Cylindrical compression simulations with Lasnex indicate that the cold fuel will be typically several hundred $\mathrm{eV}$ as compared to $10 \mathrm{keV}$ for the hot spot. Ignoring the pressure of the cold fuel we calculate the product ux using the snow plow model. The governing equation of the snow plow model is

$$
\frac{d}{d t}(\mathbf{M r})=\mathrm{PA}
$$

where $M$ is the mass of the hot spot plus the swept up cold fuel and $A$ is the area of the outer boundary of the hot spot. We assume that the fuel temperature stays constant, thus the pressure is given by $\mathrm{P}_{0} \mathrm{x}^{-2}$. This equation can be put into dimensionless form

$$
\frac{d \mathrm{u}}{d \tau}=\frac{\left(1-2 \Delta \mathrm{u}^{2} \mathrm{x}^{2}\right)}{\mathrm{x}\left(1+\Delta\left(\mathrm{x}^{2}-1\right)\right)}, \mathrm{u}=\frac{d \mathrm{x}}{d \tau},
$$

where $\tau=t / t_{0}$, and $t_{0}=\left(\rho_{0} r_{0}^{2} / 2 P_{0}\right)^{1 / 2}$, which has the asymptotic solution $\Delta(u x)^{2}=1$. The net heating is the difference between the deposited alpha power and the hydrodynamic work. We integrate the net heating rate over time, while the quantity is positive, and divide by the initial energy to obtain the net energy generated in the hot spot for heating. We divide this energy by the initial thermal energy of the hot spot and define this to be the effective hot spot gain, $G_{h}$. To obtain propagating burn the hot spot must gain enough energy to heat a layer of the cold surrounding fuel. Assuming the mass of this layer is roughly equal to the hot spot mass, we expect that $G_{h}$ must be approximately 2 . Using eqs (3)-(8) we have calculated the minimum value of $\rho_{0} r_{0}$ to achieve $G_{h}=2$ within the hot spot as a function of the ratio $\Delta=\rho_{d} / \rho_{0} \rho_{d} / \rho_{0}$, where $\rho_{c}$ is the density of the cold fuel surrounding the hot spot. The results are shown in Fig. 1 for several values of $\Gamma=r_{c \alpha} \rho / R_{\alpha}$. The parameter $\Gamma$ indicates the degree of magnetization, $\Gamma=0$ corresponds to infinite magneti- 
zation, while $\Gamma \geq 1$ corresponds to no magnetization. The results indicate that the minimum $\rho_{0} T_{0}$ is reduced by applying a magnetic field. However, the minimum value of $\rho_{0} T_{0}$ does not go to zero even in the limit of infinite magnetic field $(\Gamma=0)$. This is because the confinement time is proportional to $\rho_{0} r_{0}$. Notice that the values of $\rho_{0} x_{0}$ decrease with increasing $\Delta$, since increasing the density of the fuel surrounding the hot spot increases the confinement of the hot spot. However, since the mass of the hot spot scales as $\left(\rho_{0} r_{0} \Delta\right)^{2}$, the minimum hot spot energy occurs for $\Delta=1$.

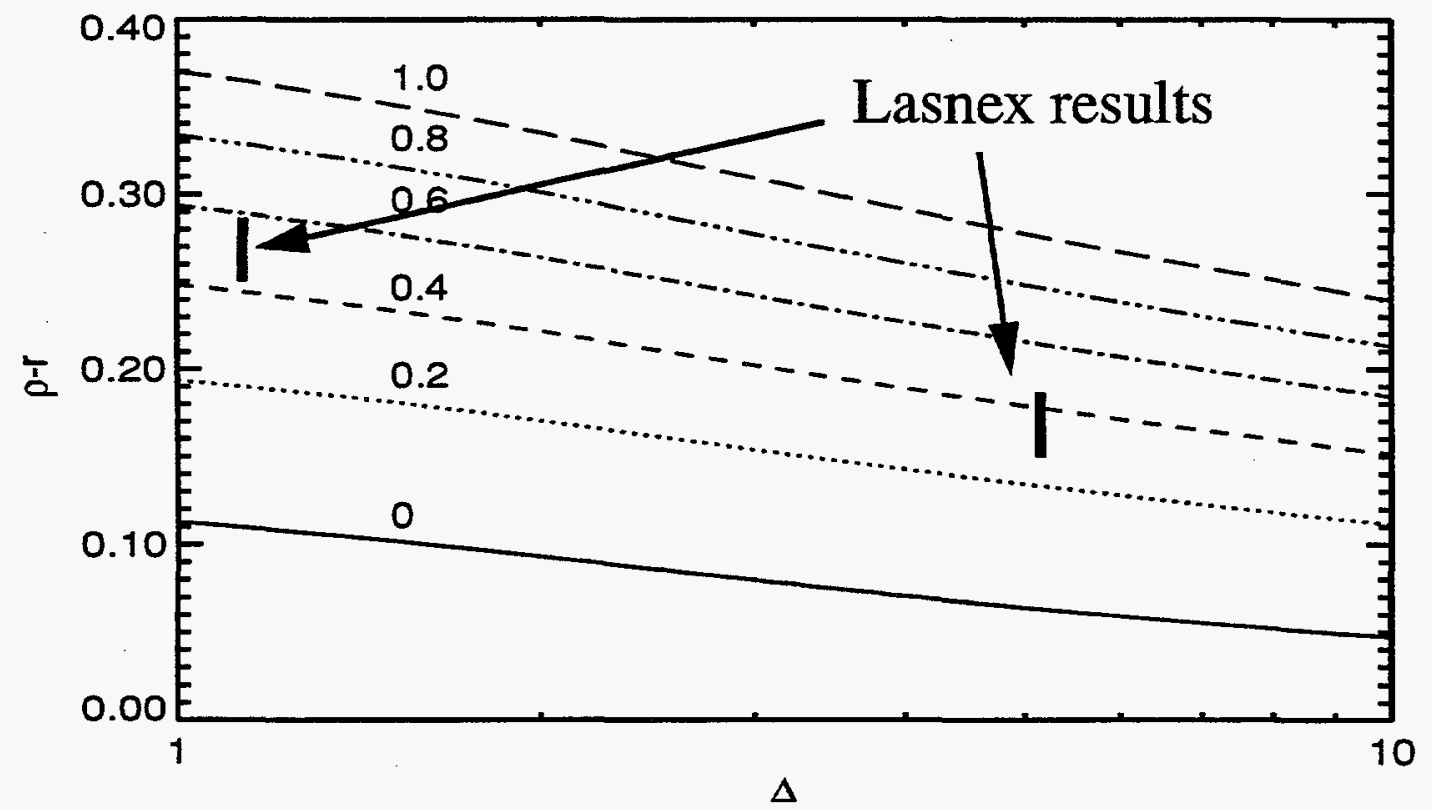

Fig. 1 The minimum $\rho_{0} \mathrm{r}_{0}$ is plotted as a function of $\Delta=\rho_{d} / \rho_{0}$, The curves are labelled with values of $\Gamma=r_{c \alpha} \rho_{0} / R_{\alpha}$.

Several 1-D cylindrical Lasnex calculations were performed to compare with this simple model. The geometry of these simulations is shown in Fig. 2. This geometry approximately represents the configuration of a liner at peak compression. For simplicity we assume that all velocities are zero at the start of these simulations. In the first sequence of simulations only the central hot spot was allowed to undergo the fusion reactions. The density of both the hot spot and the cold DT was $50 \mathrm{gm} / \mathrm{cm}^{3}(\Delta=1)$, the density of the alu- 
minum was $60 \mathrm{gm} / \mathrm{cm}^{3}$, while $\rho_{0} \mathrm{r}_{0}=0.2$ for the hot spot, $\rho_{0} \mathrm{r}_{0}=0.8$ for the cold fuel, and $\rho_{0} I_{0}=1.0$ for the aluminum pusher.

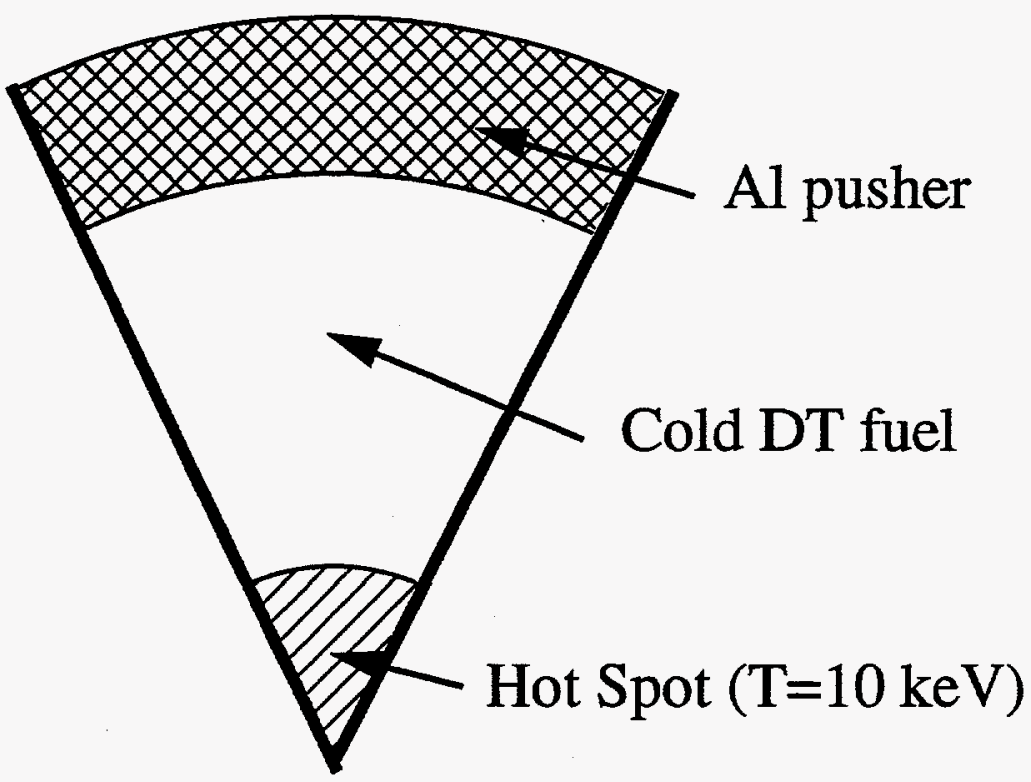

Fig. 2 Geometry of magnetized hot spot ignition simulations

Three magnetic field strengths $B=(0,225,900) \mathrm{MG}$ were used, corresponding to $\Gamma=(1.0$, $0.4,0.1)$. The gain within the hot spot as defined above was $G_{h}=(0.4,0.5,1.2)$. Clearly the magnetic field increases the hot spot gain, although these gains are lower than predicted by the simple snow plow model. The snow plow model predicted $G_{h}=2$ for $\Gamma=$ 0.2 . The simulations were repeated with fusion reactions allowed in the cold fuel region. Propagating burn did not occur in any of these simulations. A second series of simulations were performed with $\rho_{0} r_{0}=0.3$ in the hot spot, and $\rho_{0} r_{0}=0.7$. Two magnetic field strengths $B=(0,225)$ MG were used, corresponding to $\Gamma=(1.0,0.4)$, and resulting in $G_{h}$ $=(1.8,2.3)$. In both of these cases a propagating burn wave occurred in the cold fuel; however, roughly $39 \%$ of the total fuel burned for the simulation without a magnetic field, while approximately $21 \%$ of the fuel burned with the imbedded magnetic field. Appar- 
ently this is because the propagation of the burn wave was inhibited by the magnetic field. We performed further Lasnex simulations with the hot spot density at $1 / 5$ of the cold fuel density, with similar behavior. We have plotted vertical bars in Fig. 1 to indicate the $\rho_{0} r_{0}$ threshold for hot spot ignition of propagating burn into the surrounding cold fuel. The Lasnex simulations indicate that there is little benefit to magnetizing the fuel as a means of reducing the hot spot energy required to start propagating burn wave. Thus achieving high density compressions seems to be the only way to keep the hot spot energy requirement at a modest level.

\section{Quasi-spherical isentropic z-pinch compression}

The magnetic pressure is proportional to $1 / \mathrm{r}_{\mathrm{c}}{ }^{2}=1 /\left(\mathrm{r}_{\mathrm{s}} \cos (\theta)\right)^{2}$, where $\mathrm{r}_{\mathrm{c}} / \mathrm{r}_{\mathrm{s}}$ are the cylindrical/spherical radii. A quasi-spherical implosion can be obtained by tapering the liner so that the liner thickness has the form $\sigma=\sigma_{0} / \cos (\theta)^{2}$. In addition slide cones must be provided to force the fuel to compress in the axial direction as shown in Fig 2. Quasi-spherical z-pinch compression has been demonstrated at moderate pressures ${ }^{5}$.

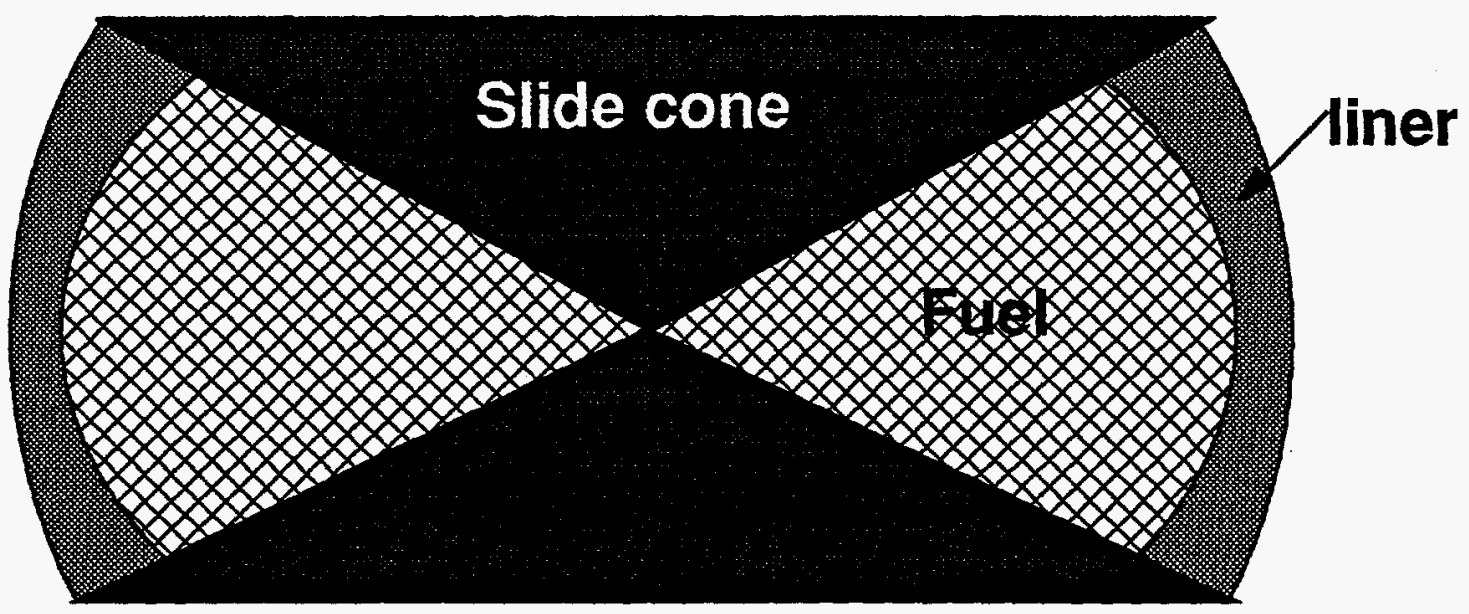

Fig. 3 A schematic of a quasi-spherical compression

However, at the high pressures needed to compress DT to a density near $300 \mathrm{gm} / \mathrm{cm}^{3}$, the slide cones will be deformed. Furthermore, there will be sheared flow at the slide cone fuel 
interface, which will probably result in turbulent mixing. Thus it would be desirable to laser heat the compressed fuel at the equator to avoid this region. Note that it should be possible to obtain quasi-spherical compression without a pusher by starting a DT capsule with an appropriate ellipsoidal shape that deforms into a sphere in reaction to the magnetic pressure. These 2-D effects are difficult to calculate with any confidence. As a first step we shall calculate the current wave form needed to drive an ideal system, i.e. fixed slide cones and no turbulent mixing at the fuel interface.

The drive current needed to obtain a given fuel density is minimized when the pressure profile results in isentropic compression. Kidder's homogeneous isentropic compression solution $^{6}$ can be used to estimate the ideal drive current profile. The result is

$$
I=\frac{I_{0}}{\left(1-\left(\frac{t}{t_{0}}\right)^{2}\right)^{3 / 4}}
$$

where $\mathrm{I}_{0}=2 \pi \mathrm{r}_{0}\left(2 \mathrm{P}_{0} / \mu_{0}\right)^{1 / 2}$, and $\mathrm{t}_{0}=\left(\rho_{0} \mathrm{r}_{0} / \mathrm{dP}_{0} / \mathrm{dr}_{0}\right)^{1 / 2}$. Numerical simulation with the 1-D hydrodynamic code Bucky ${ }^{7}$ indicates that this profile does not work very well, probably because the initial condition is not satisfied. A better profile is found to be

$$
I=\frac{I_{0}}{\left(1-\frac{t}{t_{0}}\right)^{3 / 4}}
$$

The current profile from a typical pulsed power generator does not match this very well. Typically the current rises almost linearly with time up to peak current. Simulations indicate that such a current profile does not efficiently compress the fuel because a rather 
strong shock is launched into the fuel too early in the pulse. However, an appropriate current drive profile can be obtained by magnetic flux compression as shown in Fig. 4 .

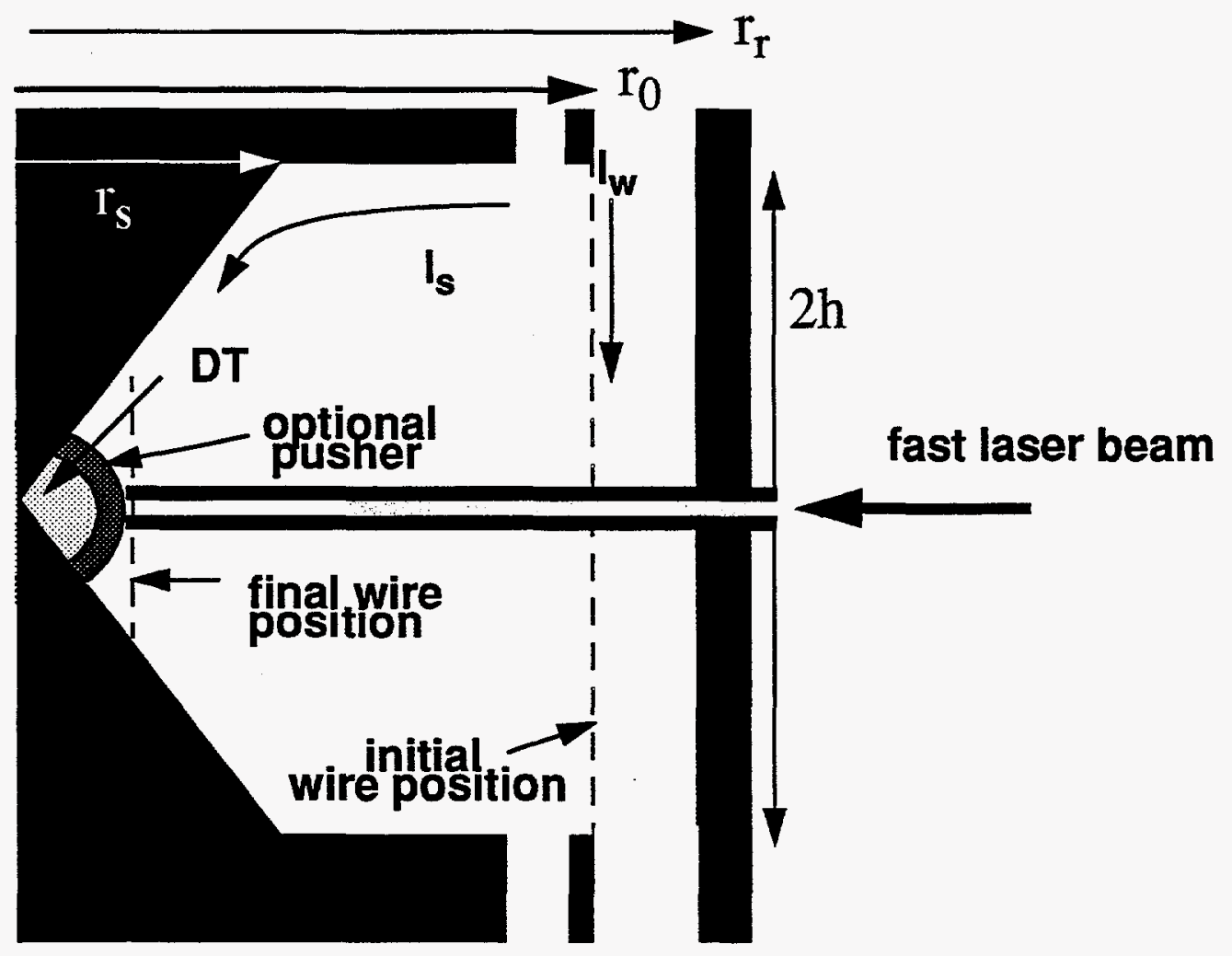

Fig. 4 Flux compression geometry showing a possible path for the fast laser beam

All of the objects in figure have cylindrical symmetry except for the fast laser which comes in a only one azimuthal angle. A seed field is generated by the current, $I_{s}$, from a feed inside the initial radius of the wire array, $\mathrm{r}_{0}$. This azimuthal field is then compressed by the wire array implosion driven by the current $I_{w}$, which returns along $r_{r} A$ laser path could be provided along the midplane or possibly along the axis (not shown). The disadvantage of having the laser come in along the axis is that there will be mixing of the slide cone material with the DT along the slip surface. Therefore the laser or the hot electrons that it produces would have to penetrate through this layer to reach uncontaminated DT. If 
a pusher can be avoided, a laser coming in along the midplane would heat clean DT directly. The equation of motion for the wire array is

$$
\frac{d^{2} \mathrm{r}}{d \mathrm{t}^{2}}=-\frac{\mu_{0} \mathrm{~h}}{4 \pi \mathrm{rM}}\left(\mathrm{I}_{\mathrm{w}}^{2}-\mathrm{I}_{\mathrm{T}}^{2}\right),
$$

where $\mathrm{I}_{\mathrm{T}}$ is the current driven through the target. Assuming that the magnetic flux is conserved between the wire array and the target, this current is given by the relation

$$
\mathrm{I}_{\mathrm{T}}=\frac{\mathrm{L}(0) \mathrm{I}_{\mathrm{s}}}{\mathrm{L}(\mathrm{t})}
$$

where $L(t)$ is the time dependent inductance of the region between the wires and the target. This inductance depends on both the motion of the wire array and the target. Since, the motion of the target involves complicated hydrodynamics, we would like to estimate this inductance without actually calculating the target radius as a function of time. Thus we divide the inductance into two parts, $\mathrm{L}(\mathrm{t})=\mathrm{L}_{1}+\mathrm{L}_{2}$. We let $\mathrm{L}_{1}$ be the inductance between the initial target radius and the final target radius and we approximate this inductance with the formula

$$
\mathrm{L}_{1} \approx \frac{\mu_{0} \mathrm{r}_{\mathrm{c}} \mathrm{h}}{\pi \mathrm{r}_{\mathrm{s}}} \ln 10
$$

where $r_{c}$ is the initial capsule radius and we have assumed a final compression ratio of 10 . Then assuming that the wire can never go to a radius smaller than the capsule radius we obtain the approximate formula

$$
L_{2}=\frac{\mu_{0}}{\pi}\left(r_{c} \ln \frac{r_{w}(t)}{r_{c}}+\int_{r_{c}}^{h} \ln \left(\frac{r_{w}(t)}{r_{E}(z)}\right) d z\right)
$$

where the electrode radius is $r_{E}(z)=r_{s} z / h$, and $r_{w}(t)$ is the time dependent radius of the wire array. The current $\mathrm{I}_{\mathrm{w}}$ can be found by solving a simple lumped circuit element model of a pulsed power accelerator, which yields the circuit equation 


$$
V(t)=I_{w}\left(R+L_{w}^{\cdot}\right)+\left(L_{M}+L_{w}\right) I_{w},
$$

where we have used circuit parameters appropriate for the $\mathrm{Z}$-accelerator ${ }^{8}, \mathrm{R}=0.12$, is the machine impedance, $\mathrm{L}_{\mathrm{M}}=14 \mathrm{nH}$ is the machine inductance, and the voltage is input in tabular form. The inductance of the wire array, $L_{w}$ depends on the shape of the electrodes, as does the inductance of the region between the wires and the target. Thus the temporal behavior of $I_{T}$ can then be modified by changing the ratio of $r_{S} / h$. Equations (11)-(15) were solved numerically. We found that $r_{8} / h \sim 1 / 2$ gave a current profile that approximates eq. (10) rather well. The resulting wave forms are shown in Fig. 5. In this calculation the seed current was delivered with a constant dI/dt up to a peak value of $2.7 \mathrm{MA}$ at $70 \mathrm{~ns}$ into the pulse. During this period of time the wire array has not moved much. The radius has decreased to $2.8 \mathrm{~cm}$ from the initial radius of $3.0 \mathrm{~cm}$. After the seed current is injected the wire array collapses on the field and drives the target current up to $30 \mathrm{MA}$. The drive current was only $10 \mathrm{MA}$, because the load was quite inductive due to the $6 \mathrm{~cm}$ height.

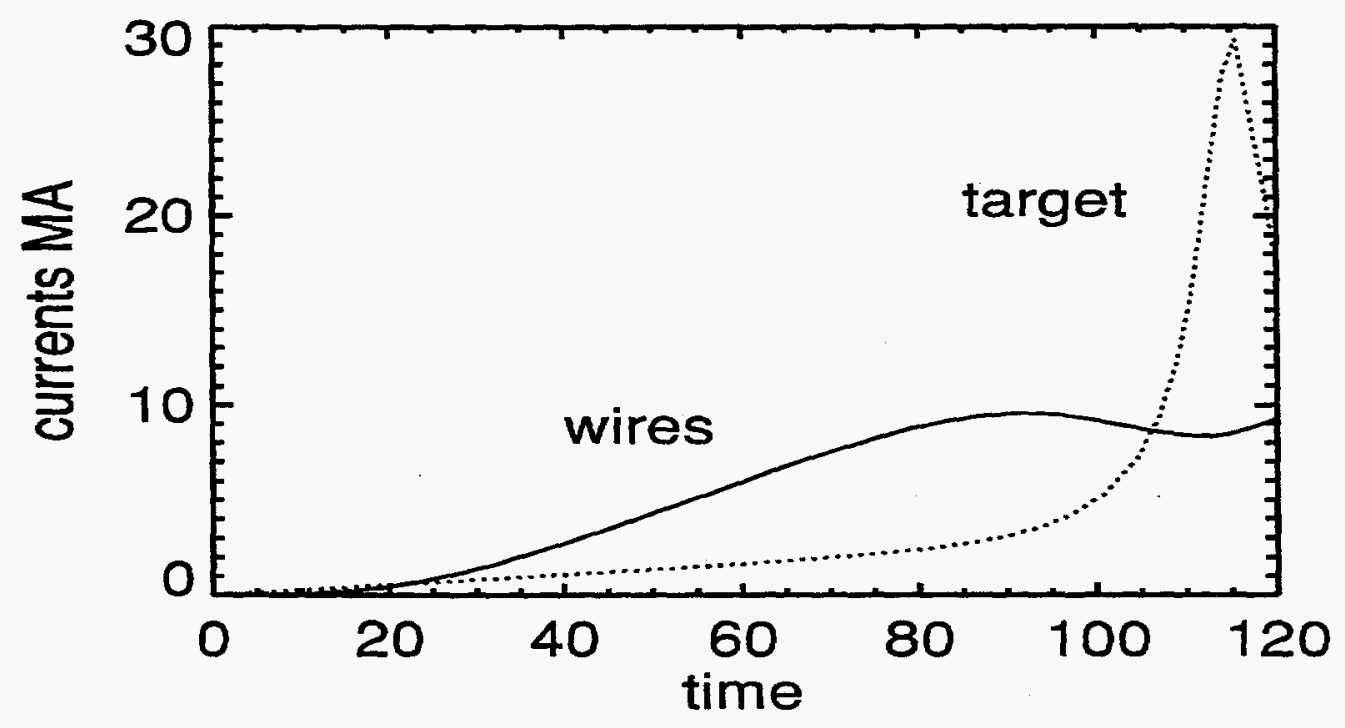

Fig. 5 Calculated current waveforms 
We used this calculated current profile delivered to the target to drive 1-D spherical Bucky $^{7}$ implosion simulations. This was implemented by a pressure boundary condition, which we set to the magnetic pressure generated by the current as given by the expression

$$
\mathrm{P}_{\mathrm{M}}=\frac{\mathrm{B}^{2}}{2 \mu_{0}}=\frac{\mu_{0} \mathrm{I}^{2}}{8 \pi^{2} \mathrm{r}^{2}}
$$

A quasi-spherical implosion by shimming a pusher, using a hollow DT sphere with a shimmed wall surface, or using a solid DT ellipsoidal shape that deforms into a sphere in reaction to the magnetic pressure. We shall not address these issues here, but will just assume a 1-D spherical compression to roughly determine the current drive needed to generate the desired compression. No pusher was used in these simulations, since using a pusher would make it more difficult to bring the ignition laser energy into the fuel. In the first simulation, a solid DT $\left(\rho=0.25 \mathrm{gm} / \mathrm{cm}^{3}\right.$ ) sphere with an outer radius of $1.5 \mathrm{~mm}$ obtained a peak fuel density of approximately $300 \mathrm{gm} / \mathrm{cm}^{2}$. The ion and electron temperature at peak compression was approximately $1 \mathrm{keV}$, which is well below ignition temperature so a fast laser would be needed to ignite the fuel. In the second simulation, the configuration consisted of a hollow solid density shell $\left(r_{\text {out }}=2 \mathrm{~mm}, r_{\text {in }}=1 \mathrm{~mm}\right)$ with an inner lower density $\left(\rho=0.025 \mathrm{gm} / \mathrm{cm}^{3}\right)$ region. The peak density in this simulation reached $600 \mathrm{gm} / \mathrm{cm} 3$, while the peak temperature in the central region reached approximately $3 \mathrm{keV}$. The $\rho$ r of the central hot region was only $0.2 \mathrm{gm} / \mathrm{cm}^{2}$. These conditions are not far from what is needed for ignition, which suggests that it might even be possible to ignite the fuel without the use of a fast laser. These calculations are encouraging, but there is important physics that has not been included. In particular, we have assumed that the magnetic flux between the wire array and the target is conserved. However, this will certainly not be rigorously true. The electrode walls, the target and the wire array plasma will have a finite resistivity and thus some of the magnetic flux will be absorbed by these surfaces. 
As a first step we have performed 1-D spherical Lasnex simulations of the flux compression process. The angle between the electrodes was made very small so the the problem is essentially $1-D$, since $\cos \theta \sim 1$. This removed the need to shim either the liner or the target. The geometry of the simulations is shown in Fig. 6

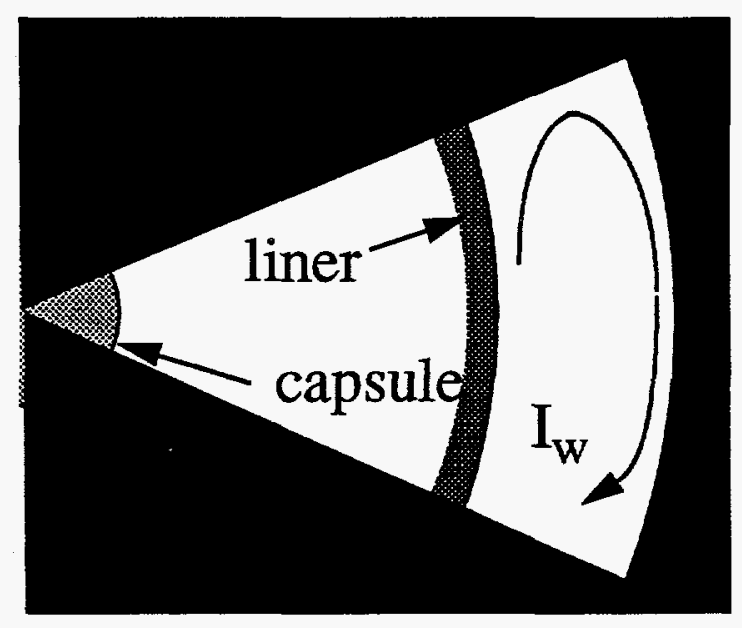

Fig. 6 Geometry for Lasnex simulations of magnetic flux compression

The Lasnex simulations were run with an exterior circuit model, which drives the current $I_{w}$. The circuit parameters were set at values consistent with the existing $\mathrm{Z}$-accelerator. The capsule was solid DT with an initial radius of $2.0 \mathrm{~mm}$, the liner plasma started at an initial radius of $2 \mathrm{~cm}$. A seed current of $2.7 \mathrm{MA}$ applied to the region between the capsule and the liner plasma resulted in the best compression. The simulations showed some magnetic flux loss due to finite resistivity of the plasmas, but the peak DT densities were nearly as high as the Bucky simulations, i.e. $\rho_{\mathrm{f}} \sim 250 \mathrm{gm} / \mathrm{cm}^{3}$ as compared to $300 \mathrm{gm} / \mathrm{cm}^{3}$ for the Bucky simulation. The peak ion temperature in these Lasnex simulations was approximately $800 \mathrm{eV}$ roughly consistent with the Bucky simulation of a solid DT capsule.

These 1-D results are encouraging, since the maximum drive current is less than can be delivered by the existing Z-accelerator and we estimate that approximately $10 \mathrm{~kJ}$ of 
laser energy would be sufficient to ignite the compressed fuel at a density of $300 \mathrm{gm} / \mathrm{cm}^{3}$. Such a laser should not be prohibitively expensive. However, we have not yet addressed the effects of Rayleigh-Taylor (RT) instabilities or the deformation of the slide cones. The Rayleigh-Taylor instability could strongly effect the penetration of the magnetic flux into plasma surfaces. The deformation of the slide cones could result in lower compressions than the 1-D calculations indicate. Furthermore, shear instabilities at the DT-cone interface will mix some of the slide cone material into the fuel. Simulations to address these effects are quite complex and are beyond the scope of this small study. We hope to tackle these numerical problems in the near future. However, we believe that experiments will be required to quantitatively determine the effects of these processes.

\section{Experimental plans and preliminary results}

A graduated sequence of experiments will be needed to determine how effective zpinch driven compression will be toward attaining the high densities needed for a modest energy fast igniter. Our first concern is the delivery of large currents (>20 MA) to the small z-pinch capsule ( $\mathrm{r} \sim 2 \mathrm{~mm}$ ), which corresponds to a current density of greater than $16 \mathrm{MA} / \mathrm{cm}$. To address this issue we planned a sequence of coaxial transmission line loads of decreasing inner and outer radius. We chose thick walled 304L stainless steel for the electrode material to minimize cost and minimize hydrodynamic motion during the 100-ns of the rising current pulse. Our first experiment delivered approximately 16.7 MA to a 6mm-diameter inner conductor and 12-mm-diameter outer conductor coaxial transmission line with a total length of $1 \mathrm{~cm}$, see Fig. 7. A conservative gap of $3 \mathrm{~mm}$ between the inner and outer conductors was used even though there is evidence from other $\mathrm{Z}$ experiments that gaps as small as $1.5 \mathrm{~mm}$ can be used. The current is measured by B-dot probes at a radius of $6.4 \mathrm{~cm}$. In addition, the pressure history at the electrode (radius $\sim 1.4 \mathrm{~cm}$ ) can be inferred from the VISAR diagnostic, which uses laser interferometry to measure the back surface velocity of an iron disk ( $0.5 \mathrm{~mm}$ thick by $3 \mathrm{~mm}$ diameter) imbedded in the anode. 
In this experiment, the peak current was expected to reach $18 \mathrm{MA}$ and, hence, generate a maximum value of surface magnetic field of $\sim 1.2 \mathrm{kT}$. The radial inward force on the center conductor at peak current is calculated to be $>5 \mathrm{Mbar}$, which is beyond the yield strength of steel. However, the wall thickness of the inner conductor is large enough that inertia keeps this electrode from moving significantly. The magnetic field strength is substantially smaller at the location of the VISAR diagnostic due to the larger radius, but the pressure is still approximately $240 \mathrm{kbar}$. At this pressure the equation of state of aluminum is known well enough to unfold the pressure history at the electrode surface from the velocity information obtained by the VISAR diagnostic.

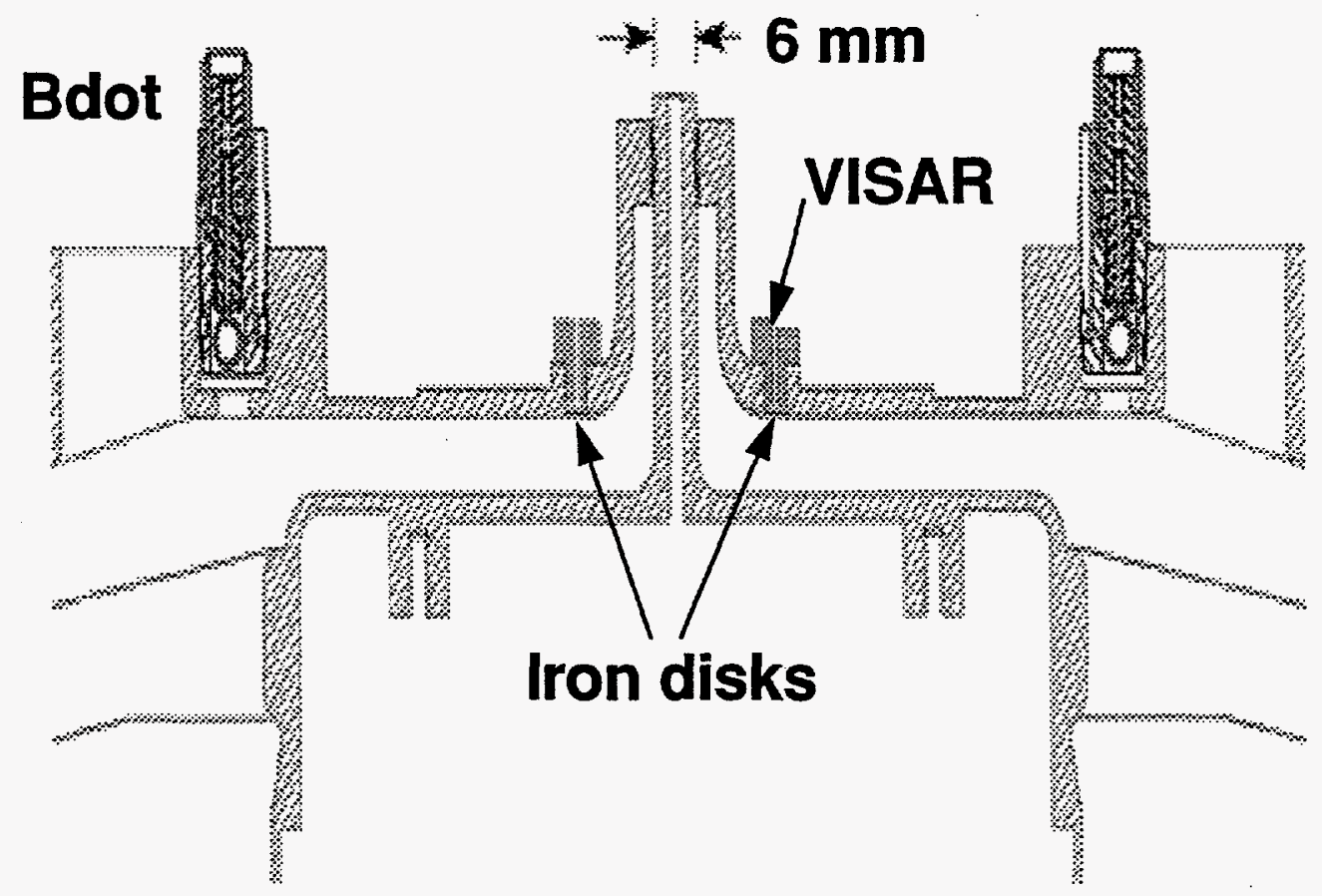

Fig. 7 A schematic cross section of the load region

Data obtained from B-dot monitors from this $\mathrm{Z}$ shot (315) show that we successfully delivered at least 16.7 MA to the short circuit coaxial load. The injected current wave form measured at the vacuum interface and the load current wave form are plotted in Fig. 8. These results show that up to $10 \mathrm{MA} / \mathrm{cm}$ can be efficiently delivered to a small z-pinch load. We plan to continue these experiments with smaller inner diameters. 


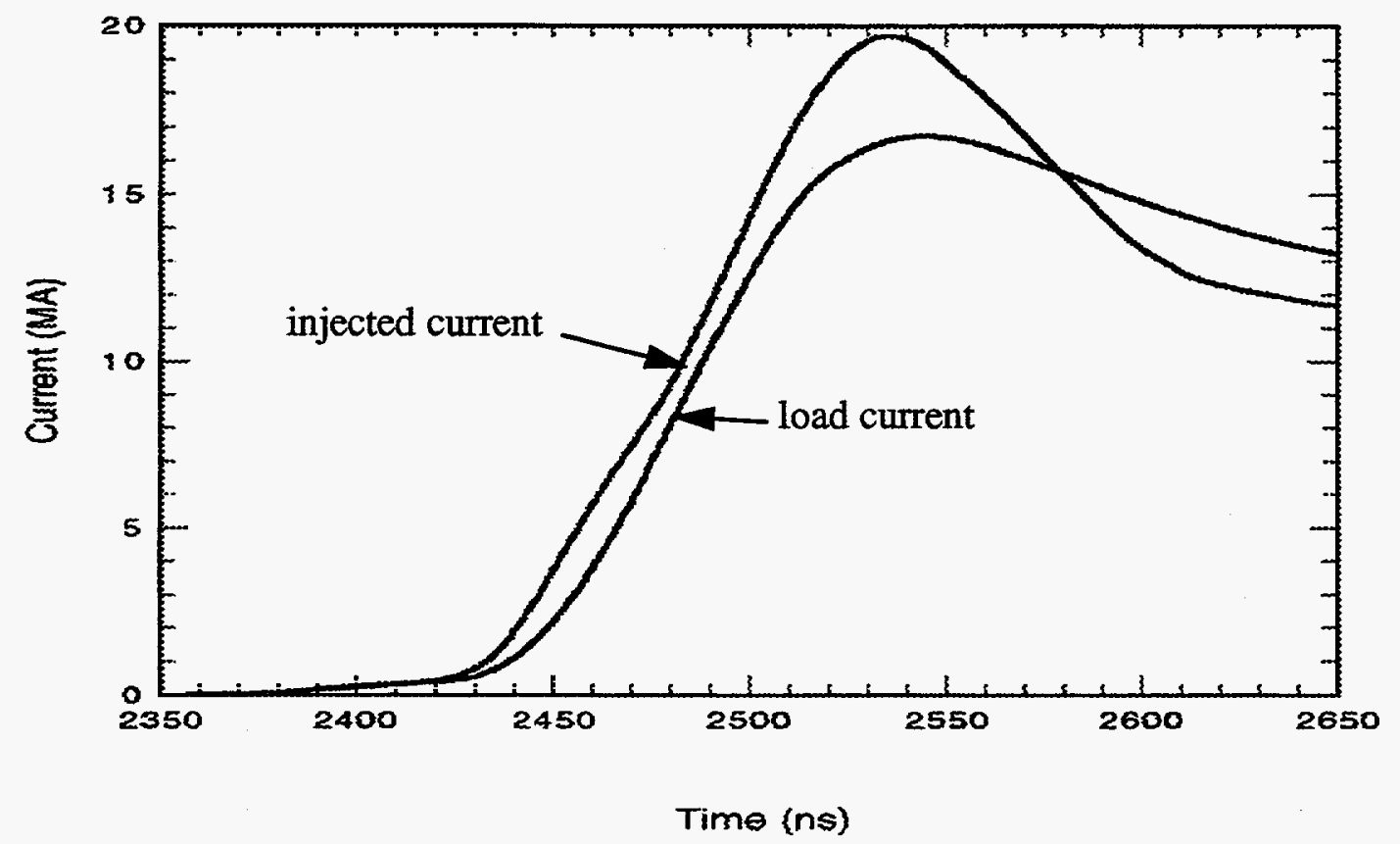

Fig. 8 Current wave forms for shot 315

We further plan to use thin walled hollow cylinders filled with foam to develop the capability to compress materials. Note that a foam of appropriate density is a convenient substitute for solid DT. Pressure measurements will be critical to determining the degree of compression that is achieved. In addition such measurements can be used to determine the equation of state (EOS) of various materials.

In developing the capability to diagnose these types of studies, techniques commonly used in conventional impact generated experiments were implemented. The primary diagnostic presently being used for this work is velocity interferometry, VISAR $^{9}$, which provides particle velocity measurements. Samples of copper and iron were placed at the electrode position labelled VISAR as shown in Fig. 7. The equation of state of these materials is known well enough to unfold the pressure history at the electrode surface from the velocity information obtained by the VISAR diagnostic ${ }^{10}$. Using the relation between 
magnetic pressure and current as given by eq. (16), we obtained a peak current of approximately $19 \mathrm{MA}$. This is in reasonably good agreement with the Bdot data.

Since the current rises roughly linearly with time this magnetic pressure does not produce a shock for most of the pressure pulse. Samples placed just beneath the electrode surface were therefore loaded isentropically over a time duration of nearly $100 \mathrm{~ns}$. The peak magnetic pressure on the samples was approximately $300 \mathrm{kbar}$. The resulting free surface particle velocity obtained on iron is shown in Fig. 9. which shows elastic yielding, the 130 kbar hcp-bcc crystallographic phase transition, and compression in the high pressure phase. These data are consistent with previous shock wave data on iron.

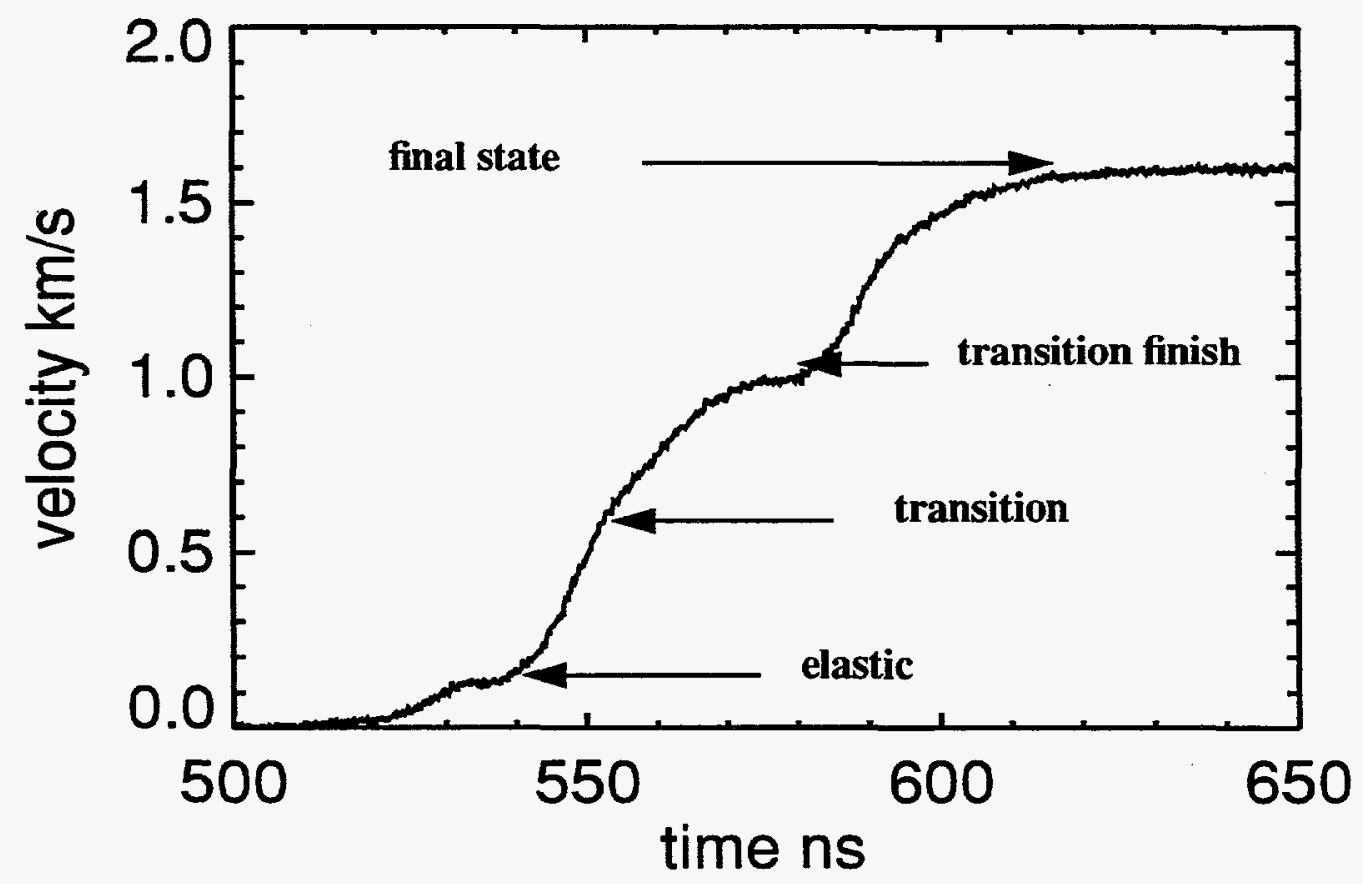

Fig. 9 VISAR velocity measurements on an Fe sample ( $0.5 \mathrm{~mm}$ thick) loaded isentropically. 
We plan to move the samples to smaller radii to determine if this technique continues to work at higher pressures. Ultimately we plan to use this technique to measure pressure profiles generated by z-pinch driven hollow cylinders compressing foam. Further down the road, we plan to introduce slide cones to obtain higher compressions and to conduct a series of experiments to study the potential of flux compression as one means to deliver high currents with short rise-times to a small diameter z-pinch load.

\section{Conclusions and future work}

We have investigated the feasibility of using z-pinch implosions to compress isotopes of hydrogen isentropically to high densities as is required for inertial fusion. This requires relatively slow pressure pulses (10-20 ns) with an appropriate time history. We found that this time history can be obtained most easily by the technique of magnetic flux compression. We also determined that quasi-spherical compression is required to obtain fuel densities high enough to seriously consider using a fast laser to ignite the fuel. We found that magnetizing the fuel did not significantly affect the fuel density requirement to obtain high gain. A series of 1-D numerical simulations indicated that DT could be compressed to high densities with a drive current which could be provided by the existing Z-accelerator. These simulations did not include 2-D effects such as the Rayleigh-Taylor instability, slide cone deformation, or shear instabilities at the fuel slide cone interface. Each of these effects could significantly reduce the fuel compression. We plan to study these effects both numerically and experimentally in the future. We have presented data from our first experiment, which indicates that current can be efficiently delivered to the small loads appropriate to this approach to inertial fusion. This experiment also allowed us to test the VISAR diagnostic approach to measuring pressures. This diagnostic will help us determine the degree of fuel compression in future experiments. 


\section{References}

1 J. Nuckolls et. al. Nature, 239, 139, 1972

2 M. Tabak, I. Hammer, M. E. Glinsky, W. L. Kruer, S. C. Wilks, J. Woodworth, E. M. Campbell, M. D. Perry, and R. J. Mason, Phys. Plasmas 1, 1626, 1994

3 A. Pukhov and J. Meyer-ter-Vehn, Phys. Rev. Lett, 79, 2686, 1997

4 G. B. Zimmerman and W. L. Kruer, Comments Plasma Phys. Controlled Thermonucl. Fusion 2, 511975.

5 Degnan et. al. Phys. Rev. Lett. 74, 98, 1995.

6 R. E. Kidder, Nuclear Fusion, 14, 797, 1974.

7 MacFarlane et. al. Univ. of Wisconsin Fusion Tech. Inst. Rep. UWFDM-984, 1995.

8 M.K. Matzen, Phys. Plasmas 4 (5), 1519 (1997).

9 L.M. Barker and R.E. Hollenbach, " Laser Interferometer for Measuring High Velocities of any Reflecting Surface ", J. Appl. Phys. 43, 4669, (1972).

10 J.R. Asay and M. Shahinpoor, High Pressure Shock Compression of Solids, New York, Springer Verlag, 1993. 


\section{distribution}

3 Comell University

Laboratory of Plasma Studies

Attn: $\quad$ D. A. Hammer

B. R. Kusse

369 Upson Hall

J. B. Greenly

Ithaca, NY 14853

5 Lawrence Livermore National Laboratory

Attn: John Perkins L-637

B. G. Logan L-481

J. Hammer L-018

John Lindl L-039

M. Tabak L-015

A. Toor L-041

P.O. Box 55117000 East Ave

Livermore, CA 94550

2 Imperial College

Attn; M. Haines

G. Chittenden

South Kensington, London

SW72BZ, United Kingdom

1 D. G. A

Attn:

J. Leon

46500, Gramat, France

1 Institute fur Kernphysik

Attn: $\quad$ D. H. H. Hoffmann

Strahlungs-und Kernphysic

TU Darmstadt, Karolinenplatz

D 64289 Darmstadt, Germany

1 Universite Paris SUD

Attn: $\quad$ C. Deutch

Laboratoire de Physique des Gas et des Plasmas

BAT 210 LP CEP R-91405

Orsay, France

1 University of Nevada Reno

Attn: $\quad$ B. Bauer

Department of Physics, Reno, NV 89557

2 Los Alamos National Laboratories

Attn: $\quad$ D. Peterson X-PA

R. Bowers X-TA

Los Alamos, NM 87545 
1 Weizman Institute of Physics

Attn: I. Maron

Department of Physics

Rehovot 76100, Israel

1 I.N.R., Forschungzentrumkarlsruhe

Attn: Hans Bluhm

Postfasche 3640

D-76021, Karlsruhe, Germany

1 Lawrence Berkeley National Laboratory

Attn: R. O. Bangerter

1 Cyclotron Rd

Berkeley, CA 94720

1 University of California San Diego

Attn: $\quad$ M. Rosenbluth

Dept. of Physics, B019 UCSD

La Jolla, CA 92093

1 University of California Davis

Attn: J. DeGroot

Dept. of Applied Science

Rm 228 Walker Hall

Davis, CA 95616

1 Polymath Associates

Attn: Bedros B. Afeyan

474 Mission Dr.

Pleasanton, CA 94566

$\begin{array}{llll}1 & \text { MS-0188 } & 4523 & \text { C.E. Meyers } \\ 1 & \text { MS-0151 } & 9000 & \text { G. Yonas } \\ 1 & \text { Ms-1190 } & 9500 & \text { D. Cook } \\ 1 & \text { MS-1188 } & 9500 & \text { C. Olson } \\ 1 & \text { MS-1191 } & 9502 & \text { J. Quintenz } \\ 1 & \text { MS-1191 } & 9502 & \text { M. Sweeney } \\ 1 & \text { MS-1181 } & 9511 & \text { J. Asay } \\ 1 & \text { MS-1181 } & 9511 & \text { C. Hall } \\ 1 & \text { MS-1181 } & 9511 & \text { K. Holland } \\ 1 & \text { MS-1188 } & 9512 & \text { R. Hamil } \\ 1 & \text { MS-1188 } & 9512 & \text { S. Cameron } \\ 1 & \text { MS-1182 } & 9516 & \text { D. Bloomquist } \\ 1 & \text { MS-1194 } & 9540 & \text { D. McDaniel } \\ 1 & \text { MS-1194 } & 9544 & \text { R. Spielman } \\ 1 & \text { MS-1194 } & 9544 & \text { C. Deeney } \\ 1 & \text { MS-1194 } & 9544 & \text { M. Douglas } \\ 1 & \text { MS-1194 } & 9544 & \text { W. Stygar }\end{array}$




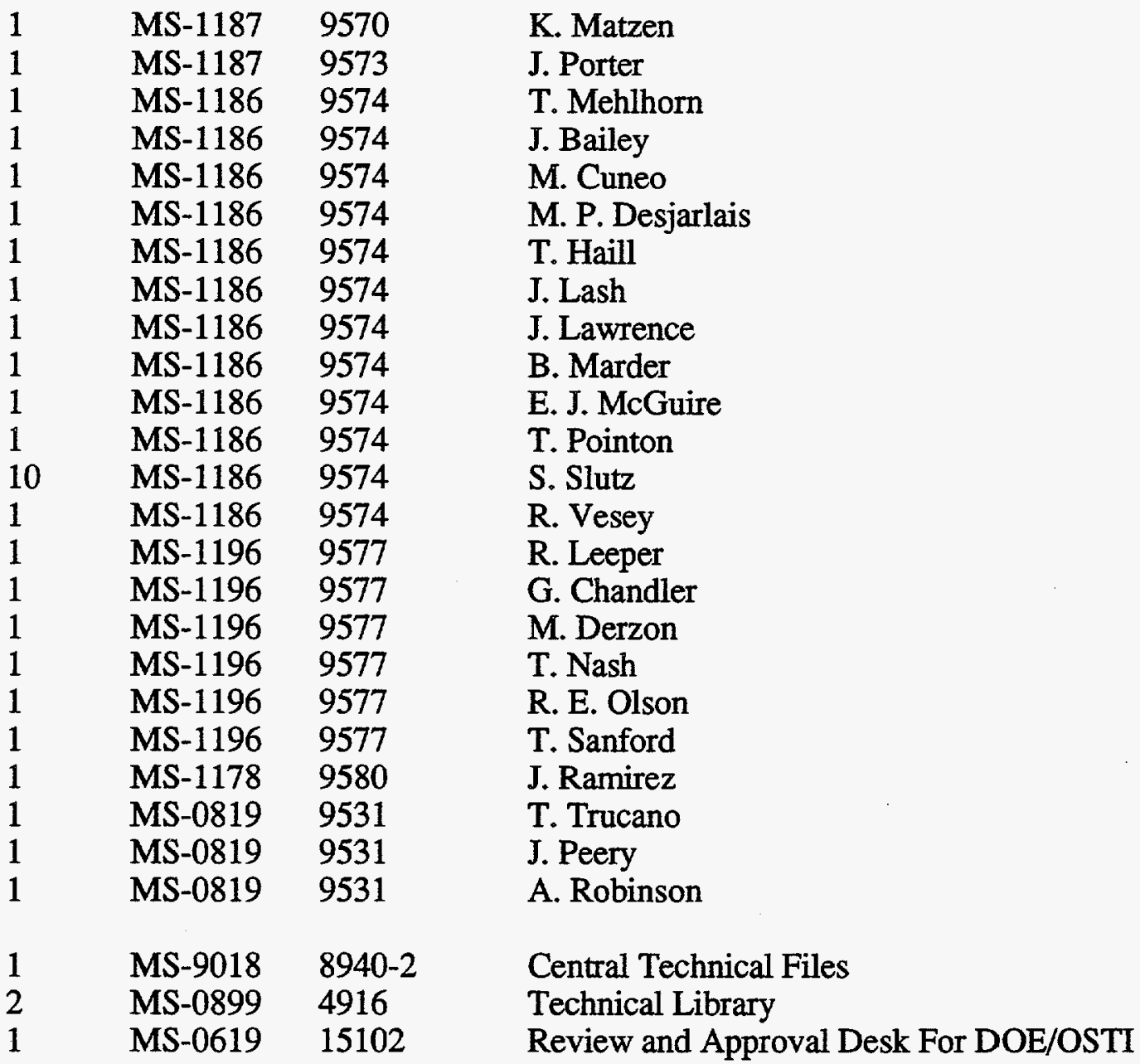

